\title{
Tianxia als Grundprinzip der globalen Gerechtigkeit? Vier Rückfragen an Zhao Tingyang
}

\section{Christian Neuhäuser, Dortmund}

Zhao Tingyang argumentiert in seinem Buch eindringlich dafür, dass ein gemeinsames Verständnis einer politisch vereinten Welt benötigt wird, um dazu befähigt zu werden, den Problemen der Menschheit entgegenzutreten. Nur so kann die Menschheit von einem Zustand des mehr oder weniger feindseligen Wettbewerbs untereinander zu einem Zustand friedlicher Kooperation gelangen. Er befürwortet eine Wiederaufnahme des antiken chinesischen Begriffs des Tianxia. Die Überlegungen in seinem Buch sind sehr aufschlussreich und leisten einen wichtigen Beitrag zur globalen Philosophie. Zhao zeigt damit auf ähnliche Weise wie Amartya Sen oder Jiwei Ci wie globale Philosophie gelingen kann. Schon hier zeigt sich, dass gegenwärtig die Impulse für eine Weltphilosophie aus Asien kommen. So bildet das Buch selbst vielleicht sogar einen notwendigen Schritt im Prozess der Herstellung eines Tianxia-Systems. Ich möchte dazu vier Kommentare abgeben.

\section{Aufklärung}

In manchen seiner Kritikpunkte zur Einseitigkeit der Moderne zeigen die Überlegungen von Zhao eine große Nähe zu Max Horkheimer und Theodor Adorno in ihrer Dialektik der Aufklärung (Horkheimer und Adorno 1944/1988). Das gilt beispielsweise für seine Kritik an unreflektiertem Konsum und dem Mangel an Spiritualität. Meiner Auffassung nach benennen Horkheimer und Adorno zwei Hauptkritikpunkte an der Aufklärung. Erstens geht mit der Verfolgung des Ziels der Ausrottung aller Ursachen von Angst die vollständige Zerstörung aller Mythen durch die Aufklärung einher. Diese Zerstörung schließt auch solche Mythen mit ein, die erhalten bleiben sollten, weil sie die Wahrnehmung schärfen für etwas, das weder kontrolliert noch verstanden oder benannt, sondern nur gefürchtet werden kann. Wenn 
dieser Sinn verloren geht, wird das Leben leer und bedeutungslos. Zweitens argumentieren sie, dass die Aufklärung lediglich eine intellektuelle Bewegung darstellt, bei welcher die materiellen Voraussetzungen und notwendigen Veränderungen sozialer Strukturen für eine wirklich gelingende Befreiung außer Acht gelassen wurden.

Dazu möchte ich folgende Fragen stellen: Erstens, benötigt Tianxia eine Art des globalen Mythos, der durch Musik und Kunst verkörpert wird (wie bei Adorno), oder reicht es aus, einen solchen Mythos auf einer eher regionalen Ebene zu haben, wenn überhaupt? Welche Maßstäbe werden benötigt, um sicherzustellen, dass die aufgeklärte Vernunft nicht in einem einseitig positivistischen Sinne rational wird? Zweitens, was genau sind die notwendigen materiellen Voraussetzungen des Tianxia? Wer würde die Erdkugel, ihre Ressourcen und das Land besitzen? Wie wären die Produktionsmittel verteilt? Wie ungleich würde das Einkommen sein?

\section{Minxin}

Zhao spricht von dem „Herzen des Volkes“ („Minxin“, übersetzt als „Volksseele“, vielleicht aber auch „Menschheitsseele“?). Es ist mir nicht klar, was genau das ist und wie dieser Zustand auf einem globalen Level erreicht werden kann. Gleichzeitig scheint Minxin entscheidend für die Realisierung des Tianxia zu sein, denn nur, wenn es Minxin gibt, ist das politische System in den Augen des Volkes legitim, im Sinne eines reflektierten Legitimitätsglaubens. An einer Stelle verknüpft Zhao den Begriff Minxin mit dem Gemeinwillen (volonté générale) - dies impliziert einen sehr starken Diskurs über kollektiv geteilte grundlegende Werte. Welche Strukturen, welche Akteure würden einen solchen Diskurs fördern? Wird die Theorie hier nicht zu idealistisch und verliert ihren realistischen Anspruch?

Zhao sagt weiter, dass Minxin ein universelles Wertesystem ist und universell akzeptiert, ja sogar beherzigt werden muss. Aber welche Werte hätten eine Chance darauf, auf globaler Ebene nicht nur rational akzeptiert, sondern von allen Akteuren beherzigt zu werden? Die Menschenrechte wären möglicherweise ein Kandidat, aber auf einem realistischen Level muss Zhao recht gegeben werden, wenn er dagegenhält, dass die gegenwärtigen Menschenrechte häufig missbraucht werden und deswegen für viele Staaten und ihre Bevölkerung inakzeptabel sind. (Allerdings vertritt Zhao eine viel zu strenge Auslegung von Rawls, denn Rawls differenziert zwischen skrupellosen Staaten und belasteten Gesellschaften.) So stellt sich die Frage: In welcher Art von Prozess kann das System der Menschenrechte ersetzt oder verändert werden, 
damit es dem Prinzip von Minxin entspricht oder sich zumindest annähert, sodass es eine solide Grundlage für Tianxia darstellen kann?

\section{Respekt}

Einvernehmlicher Respekt scheint ein wesentlicher Bestandteil von Tianxia zu sein, einschließlich der Achtung für kulturelle und religiöse Vielfalt. An dieser Stelle gibt es ein ziemlich großes Problem, denn es ist nicht klar, wie der moralische Standpunkt eines jeden Einzelnen in einem Tianxia-System bestimmt werden soll und wie umfassend dieser moralische Standpunkt sein würde. Beinhaltet dieser Standpunkt gleiche Rechte für jeden? Wenn ja, welche Rechte wären dies? Daraus entstünde eine klare Einschränkung für kulturelle und religiöse Vielfalt. Universelle Rechte stehen mit vielen kulturellen Praktiken im Widerspruch, etwa sexistischen oder rassistischen Praktiken und solchen, die Ausdruck religiöser Arroganz und kulturellen Snobismus sind.

Ich halte dieses Problem für zentral, denn ein Merkmal der Moderne ist, dass Menschen als gleichrangige Gesellschaftsmitglieder geachtet werden wollen. Hinter diese Forderung lässt sich weltweit nicht mehr zurückgehen und ein hierarchisches Modell der Achtung des klassischen Tianxia-Systems ist unrealistisch geworden. Digitale Kommunikation führt zu einer Globalisierung von Mustern des sozialen Vergleichens und Unterscheidens. Aus diesem Grund werden einzelne Staaten und erst recht familienartige Clanstrukturen nicht dazu in der Lage sein, die immer lauter werdende Forderung nach gleicher Achtung zu erfüllen. Wie stellt das Tianxia-System sicher, dass alle Menschen unter diesen Bedingungen der globalen Kommunikation die gleiche Achtung erhalten? Mir erscheint dies ein wichtiger Aspekt des Minxin zu sein, der für Tianxia notwendig ist. Aber Minxin ist zu unterbestimmt, um dies leisten zu können.

\section{Konzerne}

Zhao kritisiert an diversen Stellen des Buchs den Aufstieg der technokratischen Macht. Trotzdem erwähnt er niemals Unternehmen. Meinem Verständnis nach stellen globale Unternehmen aber einige der mächtigsten Akteure unserer Zeit dar. Mehr als zwei Drittel der 200 größten ökonomischen Einheiten der Welt sind Konzerne. Hier zeigt sich folgendes Dilemma: Unternehmen bieten ideale Strukturen für das egoistische Streben nach Profit in einem Umfeld des Wettbewerbs. Als solche scheinen sie im Widerspruch zum Tianxia-System zu stehen. Gleichzeitig wird intern versucht, in Unternehmen Familienstrukturen und gewissermaßen Minxin herzustellen: Jack 
Ma von Alibaba stellt eine perfekte Vaterfigur dar und wird auch als solche im Dream Village gefeiert.

Welche Rolle also spielen Unternehmen im Modell von Zhao? Sie lassen sich in seine Trias von Tianxia - Staat - Clan nicht so gut einordnen, es sei denn, man begreift sie als neue Clans des 21. Jahrhunderts, die allmählich übermächtig werden.

Dementsprechend treten zwei Probleme auf: Erstens, wie kann Tianxia gegen den Widerstand von Unternehmen gestärkt werden? Oder können Unternehmen so verändert werden, dass sie Tianxia befördern? Zweitens, würden globale Unternehmen in einem System von Tianxia überhaupt existieren? Wenn ja, wie wären sie strukturiert? Es scheint für sie keinen Platz in der Tianxia-Staat-Clan-Struktur zu geben. Wenn Unternehmen nicht existieren, wie würden große ökonomische Projekte dann organisiert?

\section{Zusammenfassung}

An verschiedenen Stellen sagt Zhao, dass niemand die Zukunft vorhersagen kann. Das ist mit Sicherheit wahr. Aber wir können versuchen, zukünftige gesellschaftliche Entwicklungen durch unser reflektiertes Handeln zu beeinflussen. Ich habe Tianxia als eine Möglichkeit interpretiert, die Zukunft auf eine gute Art zu verändern. Meinem Verständnis nach stellt Tianxia einen fruchtbaren Weg dar, sich globalen Prinzipien der Gerechtigkeit anzunähern, insbesondere durch die Betonung von Universalität, Kooperation und Minxin. Gleichzeitig denke ich, dass Zhao in seiner Diskusion der strukturellen Institutionen von Tianxia politischer werden sollte. Insbesondere fehlt eine Reflexion über die zentralen politischen und ökonomischen Institutionen für das Tianxia-System. Überdies sollten wir darüber diskutieren, inwiefern die Idee von Tianxia und damit verwandte Prinzipien in einem globalen philosophischen Diskurs weiterentwickelt und mit anderen, beispielsweise stärker radikaldemokratischen Ideen, verbunden werden können. Dies scheint einen zentralen Unterschied zu der altertümlichen Version von Tianxia, die nur für China galt, darzustellen. Die Entwicklung eines zeitgemäßen Tianxia bedarf einer stärkeren interkulturellen Reflexion, vor allem aber einer echten und auch einigermaßen bescheidenen Offenheit gegenüber ähnlichen Ideen der westlichen Philosophie.

\section{Literatur}

Horkheimer, Max, und Theodor Adorno. 1944/1988. Dialektik der Aufklärung: Philosophische Fragmente. Frankfurt/M.: Fischer Verlag. 